\title{
Simple resection of truncal desmoid tumors: A case series
}

\author{
YOSHIHIRO NISHIDA, SATOSHI TSUKUSHI, HIROSHI URAKAWA, SHUNSUKE HAMADA, \\ EIJI KOZAWA, KUNIHIRO IKUTA and NAOKI ISHIGURO
}

Department of Orthopedic Surgery, Nagoya University Graduate School of Medicine, Nagoya, Aichi 466-8550, Japan

Received March 9, 2015; Accepted June 7, 2016

DOI: $10.3892 / \mathrm{ol} .2016 .4792$

\begin{abstract}
Desmoid tumors of the extra-abdominal and abdominal wall have been associated with morbidity due to the aggressive nature of the surgery and high recurrence rates. Surgery that does not cause functional impairment is desired for patients with desmoid tumors. In the present study, among patients with desmoid tumors who were prospectively and consecutively treated with identical conservative treatment with meloxicam, a selected patients of patients were treated with less invasive surgery than wide-resection. Out of 60 patients pathologically diagnosed with desmoid tumors, 9 patients with tumors refractory to conservative treatment and 4 patients who refused to receive this type of treatment were treated with planned simple resection. Subsequently, the clinical outcome of the patients and the mutational status of the catenin $\beta-1$ (CTNNB1) gene in the tumors were analyzed. The mean age of the 13 patients that underwent planned simple resection was 39 years, and the tumors were located in the abdominal wall in 6 cases, the chest wall in 4 cases and the neck in 3 cases. All excised specimens were evaluated and positive microscopic margins were identified; however, during the mean follow-up period of 30 months, 12/13 cases, 7 of which had T41A mutations and 5 of which had no mutations (wild-type), did not develop recurrence. Only 1 initial case with an S45F mutation in the CTNNB1 gene developed recurrence. The results of the present prospectively treated with simple resection and retrospectively analyzed study suggest that planned simple resection could serve as a therapeutic modality for extraperitoneal desmoid tumors, particularly truncal ones with a wild-type or T41A mutational status.
\end{abstract}

\section{Introduction}

Desmoid tumor, also known as aggressive fibromatosis, is a rare, deep-seated, mesenchymal fibroblastic tumor. This type

Correspondence to: Professor Yoshihiro Nishida, Department of Orthopaedic Surgery, Nagoya University Graduate School of Medicine, 65 Tsurumai, Showa, Nagoya, Aichi 466-8550, Japan

E-mail: ynishida@med.nagoya-u.ac.jp

Key words: extraperitoneal desmoid, CTNNB1, simple resection, margin positive, meloxicam of tumor does not metastasize, but is highly locally invasive, and exhibits a propensity for recurrence, even following aggressive surgery with free surgical margin (1). Surgery has been the mainstay of treatment for desmoid tumors; however, there has been controversy about the association between the microscopic margin status and recurrence rates (2-9), and therefore, no definitive conclusion has been reached regarding the significance of the histological margin status.

A few studies have investigated the predictive value of catenin $\beta-1$ (CTNNB1) mutation, which is harbored by the majority of sporadic desmoid tumors, for the outcome of surgical treatment. The results of these studies, however, have been controversial, possibly due to the fact that they focused on retrospective cohorts with inhomogeneous treatment modalities (i.e. with or without radiotherapy) and different surgical margin statuses [microscopically negative (R0) or positive (R1) margins] (10-12). In previous studies by the present authors, patients with extraperitoneal desmoid tumors were prospectively and consecutively treated with meloxicam, a cyclooxygenase-2 (COX-2) inhibitor $(13,14)$, and a significant predictive value of CTNNB1 for this conservative treatment was demonstrated (15).

The present authors hypothesized that simple resections with positive surgical margins could be applicable to patients with truncal desmoid tumors, and that the outcomes of surgical treatment would be correlated with the mutational status of CTNNB1. In the present study, the surgical outcome in patients with extraperitoneal desmoid tumors mainly treated with meloxicam followed by planned simple resection was analyzed, and the mutational status of these tumors was evaluated.

\section{Patients and methods}

Treatment strategies for patients with extraperitoneal desmoid tumors. Until 2003, patients with extraperitoneal desmoid tumors were surgically treated with wide surgical margins; however, an unsatisfactory recurrence rate, even in cases with microscopically negative margins (9), prompted a treatment change to meloxicam, a COX-2 inhibitor, from 2003 onwards, based on the results of a study using genetically modified mice (16). Meloxicam treatment for patients with extraperitoneal desmoid tumors was approved by the Institutional Review Board of the Center for Advanced Medicine and Clinical Research of Nagoya University (Nagoya, Japan). Favorable short-term results were initially reported $(14,17)$; however, several patients subsequently exhibited resistance to meloxicam treatment (18). Since 2009, 
Table I. Demographic data of 13 patients prospectively treated with simple resection.

\begin{tabular}{lcccccc}
\hline $\begin{array}{l}\text { Age, } \\
\text { years }\end{array}$ & Gender & Tumor site & $\begin{array}{c}\text { Tumor size, } \\
\text { cm }\end{array}$ & $\begin{array}{c}\text { Follow-up } \\
\text { duration, months }\end{array}$ & Antecedent treatment & Rec \\
\hline 30 & F & Abd & 18.0 & 63 & Meloxicam & Mutation \\
19 & F & Abd & 13.0 & 54 & Meloxicam & WT \\
25 & F & Back & 5.0 & 48 & No & S45F \\
45 & M & Back & 5.0 & 38 & No & T41A \\
29 & F & Neck & 7.1 & 38 & Meloxicam & T41A \\
39 & F & Abd & 8.4 & 45 & Meloxicam & WT \\
70 & F & Neck & 8.7 & 26 & Meloxicam, Chemo & WT \\
36 & F & Neck & 4.5 & 26 & Meloxicam, Chemo & - \\
39 & F & Back & 17.0 & 14 & Meloxicam & T41A \\
35 & F & Abd & 14.0 & 13 & Meloxicam & T4 \\
40 & F & Abd & 12.0 & 10 & Meloxicam & T41A \\
62 & M & CW & 12.0 & 9 & No & WT \\
36 & F & Abd & 4.5 & 6 & WT \\
\hline
\end{tabular}

Chemotherapy consisted of methotrexate plus vinblastine. F, female; M, male; Abd, abdominal wall; CW, chest wall; Chemo, chemotherapy; Rec, recurrence; WT, wild-type.

the treatment modality was formally organized with low-dose chemotherapy (methotrexate and vinblastine) or planned simple surgery for patients with desmoid tumors refractory to or stable to meloxicam treatment. In order to select patients for planned simple resection, functional impairment should not be anticipated. These selection criteria exhibited a tendency to include truncal desmoid tumors, and exclude extremity desmoid tumors. Since 2010, the mutational status has been considered as one of the selection criteria based on the reports describing poor surgical outcome of patients harboring a tumor with S45F mutation (10-12). Informed consent for simple resection and use of anonymized samples for research was obtained from all patients in the present cohort. The study protocol, which included the analysis of the mutational status of CTNNB1, was approved by the Institutional Review Board of Nagoya University.

Mutation analysis for the CTNNBI gene. In all patients enrolled in the present study since August 2008, desmoid tumors were histologically diagnosed using specimens obtained by incisional biopsy at the time of referral and treated at Nagoya University Hospital. Part of the obtained specimens were snap-frozen and stored at $-80^{\circ} \mathrm{C}$ for mutation analysis as previously described (15). Briefly, DNA was extracted from the frozen tissue or 5- $\mu \mathrm{m}$-thick formalin-fixed, paraffin-embedded tissue using the High Pure PCR Template Preparation kit (Roche Diagnostics GmbH, Mannheim, Germany), according to the manufacturer's protocol. The extracted DNA was subjected to polymerase chain reaction (PCR) analysis in order to determine the existence of point mutations in codons 41 or 45 of CTNNB1 exon 3 using two pairs of primers spanning these mutation sites, as previously described (15). Amplified PCR products were subjected to direct sequencing. All sequencing results were compared with $\beta$-catenin sequences in the National Center for Biotechnology Information databases using The Basic Local Alignment Search Tool to evaluate the mutational status of the CTNNB1 gene.
Planned simple resection. Simple resection was planned to minimize the extent of resection of surrounding normal tissue, including muscles, fascia and other connective tissues, which reduced the rate of soft tissue reconstruction. No major nerve injury occurred as a result of simple resection in the present study. Desmoid tumors were excised without the cuff of surrounding tissues. Muscles were partially excised along with the tumors when detachment proved challenging. Excised specimens were all subjected to pathological evaluation. Two-directional surfaces through the midline of the excised specimens were examined for microscopic margin status by experienced pathologists. Patients with planned simple resection were routinely evaluated for local recurrence with computed tomography (CT) and/or magnetic resonance imaging (MRI) every 3 months until 1 year after surgery, and every 6 months thereafter.

Statistical analysis. Data were analyzed using the Fisher's exact test for dichotomous variables to determine correlations between the mutational status of CTNNB1 and clinicopathological characteristics. Continuous variables of age and tumor size were compared between the two groups using the Student's $t$ test. All statistical analyses were performed using SPSS version 20 (IBM SPSS, Armonk, NY, USA). P<0.05 was considered to indicate a statistically significant difference.

\section{Results}

Patient demographics. Since 2003, there were 60 consecutive cases with desmoid tumors of the abdominal or extra-abdominal wall histologically diagnosed histologically diagnosed at Nagoya University Hospital, Nagoya Memorial Hospital, and Konan Kosei Hospital. Patients treated with meloxicam (10 mg/body daily) received MRI and/or CT follow-ups at the outpatient unit of the Department of Orthopedic Surgery of Nagoya University Graduate School of Medicine (Nagoya, 
A

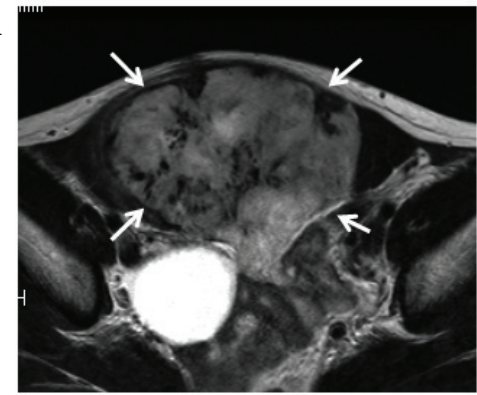

C

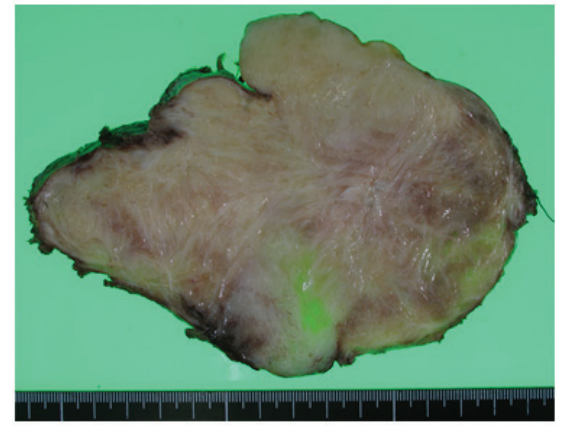

B

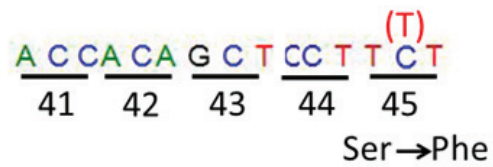

S45F
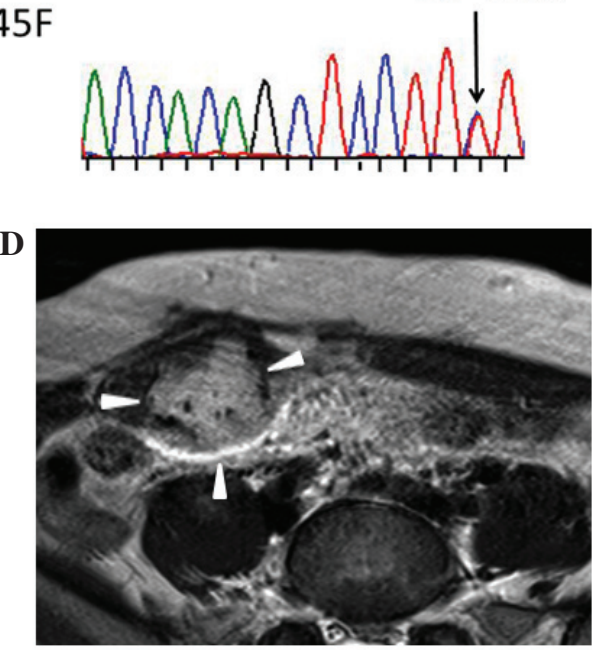

Figure 1. A 19-year-old female patient presented with a desmoid tumor in the abdominal wall. (A) A T2-weighted axial magnetic resonance image indicated the presence of a desmoid tumor (arrows). (B) CTNNB1 genotyping revealed that the tumor had an S45F mutation in exon 3. (C) The sagittal and axial surfaces of the resected tumor were subjected to histological examination. The surgical margin was R1. (D) Desmoid tumor recurrence (arrow heads) was confirmed using T2-weighted axial magnetic resonance image 16 months after R1 resection. R1, microscopically positive.

A

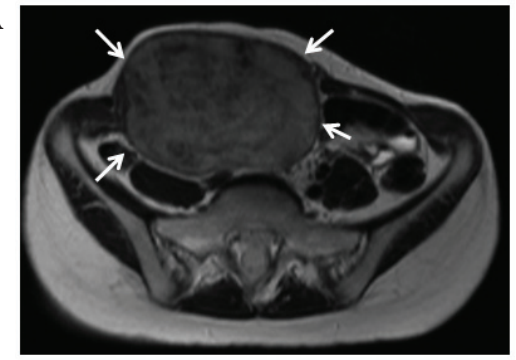

C

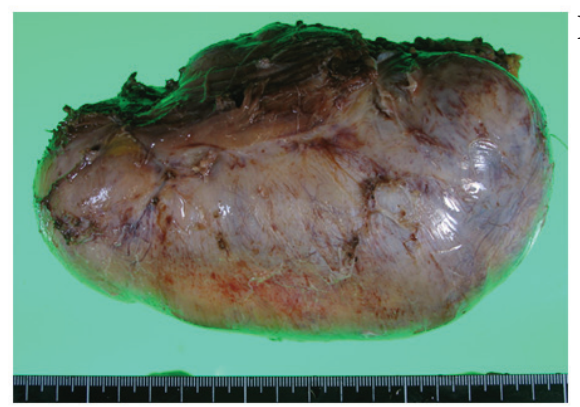

\section{$\frac{A C C A C A}{41} \frac{G C T}{43} \frac{C C T}{44} \frac{T C T}{45}$}

\section{Wild type}
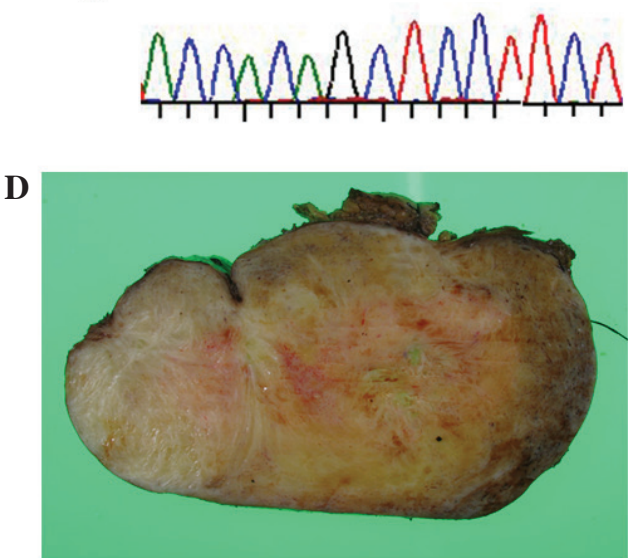

Figure 2. A 30-year-old female patient presented with a desmoid tumor in the right abdominal wall. (A) A T2-weighted axial magnetic resonance image indicated the presence of a desmoid tumor (arrows). (B) CTNNB1 genotyping revealed that the tumor was wild-type in exon 3. (C) Resected desmoid tumor. (D) The sagittal and axial surfaces of the resected tumor were subjected to histological examination.

Japan) every 3-6 months. Since 2009, 9 patients with progressive disease (PD) who received meloxicam treatment and 4 who refused to receive meloxicam treatment were subjected to planned simple resection, according to the Response Evaluation Criteria in Solid Tumors. In total. 2 out of the 9 patients with PD were initially treated with methotrexate and vinblastine, followed by planned simple resection. Of the 13 patients, 11 were female and 2 were male, with a mean age of 39 years (age range, 19-70 years). A total of 6 tumors occurred in the abdominal wall, 4 in the chest wall, 2 in the posterior and 1 in the anterior neck region. The mean diameter of the tumors was $9.9 \mathrm{~cm}$ (range, 4.5-18.0 cm). The mean and median follow-up periods after planned simple resection was 30 and 26 months, respectively (range, 6-63 months; Table I).

Mutational status of the CTNNB1 gene. Point mutations of CTNNB1 exon 3 were confirmed in $7 / 13$ cases $(54 \%)$, and 2 patterns of mutations were identified. Replacement 
of threonine by alanine (T41A) in codon 41 was detected in 6 cases, and replacement of serine by phenylalanine $(\mathrm{S} 45 \mathrm{~F})$ in codon 45 was detected in 1 case (Table I). No mutation (wild-type) was detected in exon 3 in 3/6 desmoid tumors arising in the abdominal wall, while codon 41 mutation (T41A) was detected in 2 tumors and codon 45 mutation (S45F) in 1 tumor. No significant differences were observed in the tumor site $(\mathrm{P}=0.9100)$, size $(\mathrm{P}=0.7600)$, gender $(\mathrm{P}=1.000)$ or age $(\mathrm{P}=0.9700)$ between the wild-type and mutation groups.

Outcome of surgical treatment and mutational status. All 13 patients underwent simple desmoid tumor excision. Soft tissue reconstruction following resection was required in 2/13 cases. The desmoid tumor site in both cases was the abdominal wall, and a small portion of an iliotibial band was used to patch a rectus sheath defect. No patients had surgery-related complications. Histological examination of the excised specimens revealed the surgical margin to be microscopically positive in all 13 cases. Only $1 / 13$ cases $(8 \%)$ presented with recurrence 16 months after surgery, and this case was characterized by an S45F mutation (Fig. 1). The other 12 cases, which did not present with recurrence, had a T41A mutation or had no mutation (wild-type) (Fig. 2). There was a trend of recurrence in patients with $\mathrm{S} 45 \mathrm{~F}$ mutation $(\mathrm{P}=0.077)$.

\section{Discussion}

The present study demonstrated the feasibility of simple resection in a cohort of truncal desmoid tumors prospectively treated with meloxicam. Of note, although tumor sites of the present cohort were limited in the trunk region (abdominal wall, chest wall and neck), 12 tumors with either T41A or no mutations (wild-type) did not recur with microscopic positive margins. Several recent studies investigated the association between CTNNB1 mutational status and clinical outcome of surgery (10-12,19-21). Lazar et al revealed with a single institution-based study that desmoid tumors with an S45F mutation had a worse recurrence-free survival following surgical treatment than patients with other mutations (11). Subsequently, Colombo et al reported their multicenter retrospective study (10), including Lazar's study cohort. The results of that study, which was based on 179 cases that received surgical treatment, indicated that tumors with an S45F mutation had a significantly higher recurrence rate compared with those with other mutations or wild-type tumors; however, of the 166 patients with a margin status that could be evaluated, $98(59 \%)$ had microscopically negative (R0) resection and 68 (41\%) had microscopically positive (R1) resection, indicating that the margin status was not identical in their cohort, making it difficult to draw any definitive conclusions regarding the association between recurrence and mutational status and/or margin status. Contrary to the results of the above studies, Mullen et al reported a slightly worse 5-year recurrence-free survival for patients with CTNNB1 mutated tumors (58\%) than for those with wild-type tumors in 115 cases treated with curative-intent surgical resection (12). In that study, radiation therapy was delivered in an adjuvant manner at the discretion of the surgeon and radiation oncologist when a high risk of recurrence was predicted on clinical grounds, which may have masked the correlation of the mutational status of CTNNB1 with local recurrence. Dômont et al (20) demonstrated a significant correlation $(\mathrm{P}=0.02)$ between higher risk of recurrence and CTNNB1-mutated tumors; however, no significant differences were observed among mutation types. Of note, after analyzing patients with R0 resection, it was observed that the recurrence rate was significantly higher in patients with mutated tumors than in those with wild-type tumors $(\mathrm{P}=0.02)(20)$. Considering that $\mathrm{R} 0$ resection appears to be more associated with functional impairment in patients with desmoid tumors, the significance of a specific genotype, including wild-type, in patients with $\mathrm{R} 1$ resection should be investigated. The present study could suggest the possible favorable prognostic value of wild-type and T41A mutation in patients with $\mathrm{R} 1$ resection.

The present study, however, had certain limitations. Thus, although prospectively treated patients with identical cohort (microscopic positive margins and no radiotherapy) were included in the study, only a small number of cases could be enrolled. Desmoid tumors arising in the extremities were not included in the present study, since a previous study had indicated a significantly higher incidence of the S45F mutation in desmoid tumors of the extremities $(\mathrm{P}=0.005)$ than in tumors of other sites (22), and simple microscopic positive resection could not be applied for the majority of patients with desmoid tumors of the extremities.

In conclusion, the present study reported a case series of successful planned simple resection even in cases with microscopic positive margins, which aimed at reducing functional impairment in patients with truncal desmoid tumors with wild-type or T41A-mutated tumors. Accumulating larger numbers of patients will help to clarify the significance of the results of the present study more precisely with prospectively treated cohorts.

\section{Acknowledgements}

The authors would like to thank Ms. Eri Ishihara for handling the study grant and purchasing experimental reagents, and Dr Eisuke Arai for supporting the study by storing tumor specimens in our department at Nagoya University Hospital. The present study was partly funded by the Ministry of Education, Culture, Sports, Science and Technology of Japan [Tokyo, Japan; grant-in-aid no. 262933341 for Scientific Research (B)] and Health Labour Sciences Research Grant of Japan (grant no. H26-014).

\section{References}

1. Reitamo JJ, Scheinin TM and Häyry P: The desmoid syndrome. New aspects in the cause, pathogenesis and treatment of the desmoid tumor. Am J Surg 151: 230-237, 1986.

2. Ballo MT, Zagars GK, Pollack A, Pisters PW and Pollack RA: Desmoid tumor: Prognostic factors and outcome after surgery, radiation therapy, or combined surgery and radiation therapy. J Clin Oncol 17: 158-167, 1999.

3. Gronchi A, Casali PG, Mariani L, Lo Vullo S, Colecchia M, Lozza L, Bertulli R, Fiore M, Olmi P, Santinami M and Rosai J: Quality of surgery and outcome in extra-abdominal aggressive fibromatosis: A series of patients surgically treated at a single institution. J Clin Oncol 21: 1390-1397, 2003.

4. Huang K, Fu H, Shi YQ, Zhou Y and Du CY: Prognostic factors for extra-abdominal and abdominal wall desmoids: A 20-year experience at a single institution. J Surg Oncol 100: 563-569, 2009. 
5. Lev D, Kotilingam D, Wei C, Ballo MT, Zagars GK Pisters PW, Lazar AA, Patel SR, Benjamin RS and Pollock RE: Optimizing treatment of desmoid tumors. J Clin Oncol 25: 1785-1791, 2007.

6. Merchant NB, Lewis JJ, Woodruff JM, Leung DH and Brennan MF: Extremity and trunk desmoid tumors: A multifactorial analysis of outcome. Cancer 86: 2045-2052, 1999.

7. Nuyttens JJ, Rust PF, Thomas CR Jr and Turrisi AT III: Surgery versus radiation therapy for patients with aggressive fibromatosis or desmoid tumors: A comparative review of 22 articles. Cancer 88: 1517-1523, 2000.

8. Salas S, Dufresne A, Bui B, Blay JY, Terrier P, Ranchere-Vince D, Bonvalot S, Stoeckle E, Guillou L, Le Cesne A, et al: Prognostic factors influencing progression-free survival determined from a series of sporadic desmoid tumors: A wait-and-see policy according to tumor presentation. J Clin Oncol 29: 3553-3558, 2011

9. Shido Y, Nishida Y, Nakashima H, Katagiri H, Sugiura H Yamada Y and Ishiguro N: Surgical treatment for local control of extremity and trunk desmoid tumors. Arch Orthop Trauma Surg 129: 929-933, 2009.

10. Colombo C, Miceli R, Lazar AJ, Perrone F, Pollock RE, Le Cesne A, Hartgrink HH, Cleton-Jansen AM, Domont J, Bovée JV, et al: CTNNB1 45F mutation is a molecular prognosticator of increased postoperative primary desmoid tumor recurrence: An independent, multicenter validation study. Cancer 119: 3696-3702, 2013.

11. Lazar AJ, Tuvin D, Hajibashi S, Habeeb S, Bolshakov S, Mayordomo-Aranda E, Warneke CL, Lopez-Terrada D, Pollock RE and Lev D: Specific mutations in the beta-catenin gene (CTNNB1) correlate with local recurrence in sporadic desmoid tumors. Am J Pathol 173: 1518-1527, 2008.

12. Mullen JT, DeLaney TF, Rosenberg AE, Le L, Iafrate AJ, Kobayashi W, Szymonifka J, Yeap BY, Chen YL, Harmon DC, et al: $\beta$-Catenin mutation status and outcomes in sporadic desmoid tumors. Oncologist 18: 1043-1049, 2013.

13. Nishida Y, Tsukushi S, Shido Y, Urakawa H, Arai E and Ishiguro N: Transition of treatment for patients with extra-abdominal desmoid tumors: Nagoya university modality. Cancers (Basel) 4: 88-99, 2012.
14. Nishida Y, Tsukushi S, Shido Y, Wasa J, Ishiguro N and Yamada Y: Successful treatment with meloxicam, a cyclooxygenase-2 inhibitor, of patients with extra-abdominal desmoid tumors: A pilot study. J Clin Oncol 28: e107-e109, 2010.

15. Hamada S, Futamura N, Ikuta K, Urakawa H, Kozawa E, Ishiguro $\mathrm{N}$ and Nishida Y: CTNNB1 S45F mutation predicts poor efficacy of meloxicam treatment for desmoid tumors: A pilot study. PLoS One 9: e96391, 2014.

16. Poon R, Smits R, Li C, Jagmohan-Changur S, Kong M, Cheon S, Yu C, Fodde R and Alman BA: Cyclooxygenase-two (COX-2) modulates proliferation in aggressive fibromatosis (desmoid tumor). Oncogene 20: 451-460, 2001.

17. Nishida Y, Tsukushi S, Urakawa H, Arai E and Ishiguro N: Is it possible to identify clinically useful prognostic groups for patients with desmoid tumors? J Clin Oncol 30: 1390; author reply 1391, 2012.

18. Hamada S, Urakawa H, Kozawa E, Futamura N, Ikuta K, Shimoyama Y, Nakamura S, Ishiguro N and Nishida Y: Nuclear expression of $\beta$-catenin predicts the efficacy of meloxicam treatment for patients with sporadic desmoid tumors. Tumour Biol 35: 4561-4566, 2014.

19. Bo N, Wang D, Wu B, Chen L and Ruixue Ma: Analysis of $\beta$-catenin expression and exon 3 mutations in pediatric sporadic aggressive fibromatosis. Pediatr Dev Pathol 15: 173-178, 2012.

20. Dômont J, Salas S, Lacroix L, Brouste V, Saulnier P, Terrier P, Ranchère D, Neuville A, Leroux A, Guillou L, et al: High frequency of beta-catenin heterozygous mutations in extra-abdominal fibromatosis: A potential molecular tool for disease management. Br J Cancer 102: 1032-1036, 2010.

21. Huss S, Nehles J, Binot E, Wardelmann E, Mittler J, Kleine MA, Künstlinger H, Hartmann W, Hohenberger P, Merkelbach-Bruse S, et al: $\beta$-catenin (CTNNB1) mutations and clinicopathological features of mesenteric desmoid-type fibromatosis. Histopathology 62: 294-304, 2013.

22. Le Guellec S, Soubeyran I, Rochaix P, Filleron T, Neuville A, Hostein I and Coindre JM: CTNNB1 mutation analysis is a useful tool for the diagnosis of desmoid tumors: A study of 260 desmoid tumors and 191 potential morphologic mimics. Mod Pathol 25: 1551-1558, 2012. 